\title{
Microwave-assisted synthesis and characterization of silver nanowires by polyol process
}

\author{
Dinesh Kumar • Kavita $\cdot$ Karamjit Singh • \\ Veena Verma $\cdot$ H. S. Bhatti
}

Received: 12 November 2014/Accepted: 27 November 2014/Published online: 10 December 2014

(C) The Author(s) 2014. This article is published with open access at Springerlink.com

\begin{abstract}
Silver nanowires have been synthesized by the polyol process with ethylene glycol as a reducing agent and polyvinylpyrrolidone as a stabilizer, using microwave technique. Crystallographic, topographic, and morphological characterizations of the synthesized nanostructures have been studied via powder X-ray diffraction and electron microscopy (Field Emission Scanning Electron Microscope) and Transmission Electron Microscope, respectively. Fourier Transform-Infrared Spectroscopy, UV-Vis absorption spectroscopy, Raman spectroscopy, Thermogravometric analysis, and X-ray photoelectron spectroscopy have been carried out for the detailed quantitative and qualitative analyses. Optical characterizations of the synthesized silver nanowires have been concluded via energy-resolved and time-resolved photoluminescence and energy dispersive X-ray spectroscopic studies, respectively. Spectroscopic studies confirm the formation of good-quality silver nanowires. The X-ray photoelectron spectroscopy investigation and Raman spectra further show that the PVP molecules are adsorbed on the surface of Ag
\end{abstract}

D. Kumar $(\bowtie) \cdot$ K. Singh · H. S. Bhatti

Department of Physics, Punjabi University, Patiala 147002,

Punjab, India

e-mail: dineshk_2@yahoo.co.in

H. S. Bhatti

e-mail: drhsbhatti03@yahoo.co.in

Kavita

Department of Physics, M M Modi College, Patiala 147001,

Punjab, India

V. Verma

Department of Physics, Govt. College Naya Nangal,

Distt. Ropar 140126, Punjab, India nanowires through $\mathrm{Ag}$ : $\mathrm{O}$ coordination. A possible growth mechanism of the $\mathrm{Ag}$ nanowires has been proposed. It is implied that the PVP molecules are used as both a protecting agent and a structure-directing agent for the growth of Ag nanowires. These studies confirm the formation of high-quality silver nanowires. Topographic and morphological studies confirm that average grain size of silver nanowires is in the nanometer range.

Keywords Silver nanowires - Polyvinylpyrrolidone microwave heating Polyol method

\section{Introduction}

Among all the nanowire materials, the synthesis of silver nanowires has been and continues to be an area of active research because of their wide applications in catalysts, scanning probes, and various kinds of electronic and photonic nanodevices. To realize the above applications, it is very important to develop a simple method for producing highly crystalline silver nanowires (AgNWs). Several approaches such as polyol process (Sun et al. 2002a), wet chemical synthesis (Kim et al. 2007), hydrothermal (Xu et al. 2006), template-guided synthesis (Sun et al. 2005), ultraviolet irradiation photoreduction (Zou et al. 2004), and electrochemical methods (Mazur et al. 2004) were used for the synthesis of AgNWs by previous researchers. Gao et. al. 2005b synthesized silver nanowires with pentagonal cross sections which have been synthesized by polyvinylpyrrolidone (PVP)-assisted polyol reduction in the presence of Pt nanoparticle seeds. The well-defined silver nanowires with five-twinned structure have been prepared by polyol reduction in the presence of poly (vinylpyrrolidone) (PVP K30-40000) (Gao et al. 2004). Among them, 
microwave-assisted polyol method is a promising route for the rapid preparation of metallic nanomaterials (Yang et al. 2013; Tsuiji et al. 2005). When microwave was irradiated into the mixture of $\mathrm{AgNO}_{3} / \mathrm{NaCl} / \mathrm{PVP}$ in ethylene glycol solution, anisotropic $\mathrm{Ag}$ nanarods and nanowires were produced preferentially. In this study, the dependences of shapes and sizes of silver nanowires and nanorods were examined on concentrations of PVP, $\mathrm{NaCl}, \mathrm{AgNO}_{3}$, and heating time.

\section{Experimental}

A microwave oven was modified by installing a condenser through the holes of the ceiling. Polyvinyl pyrrolidone (PVP, $M_{\mathrm{w}}=360 \mathrm{~K}$ Sigma Aldrich, $99.9 \%$ ) was homogeneously dispersed into ethylene glycol (EG,) in a 100-ml Pyrex beaker. Then, silver nitrate $\left(\mathrm{AgNO}_{3}\right)$ sodium chloride $(\mathrm{NaCl})$ were added into the solution and stirred until the $\mathrm{AgNO}_{3}$ and $\mathrm{NaCl}$ were dissolved completely. The resulting solutions were heated in the microwave oven at $400 \mathrm{~W}$ using the following concentrations of $\mathrm{AgNO}_{3}$ (60 $\mathrm{mM}), \mathrm{PVP}(50 \mathrm{mM})$, and $\mathrm{NaCl}(3 \mathrm{mM})$; and heating time $(3.5 \mathrm{~min})$. After $3.5 \mathrm{~min}, \mathrm{Ag}^{+}$reduction occurred completely. The final product was washed first with acetone to remove EG and the excess surfactant PVP and then multiple times with deionized water, following the washcentrifuge-redisperse purification cycle to remove small, quasi-spherical Ag nanoparticles. After removing both EG and PVP, we characterized the sample with following techniques.

\section{Characterization}

Synthesized AgNWs were characterized by powder X-ray diffraction (XRD) (Rigaku Japan, Miniflex 600), scanning electron microscopy (SEM) (JEOL Japan JSM 6510LV), field emission scanning electron microscope (FESEM) and Energy dispersive spectra (EDS) (SUPRA 55 OX-FORD INSTRUMENTS), Transmission Electron Microscope (TEM) [Hitachi (H-7500)], Fourier transform infrared spectroscopy (FTIR) (Perkin Elmer-Spectrum RX-IFTIR), UV-Vis. absorption spectroscopy (Hitachi-330 spectrophotometer), Micro-Raman spectroscopy ("Renishaw in Via Raman microscope," using $\mathrm{Ar}^{+}$laser as an excitation source with $514 \mathrm{~nm}$ line), $\mathrm{X}$ ray photoelectron spectroscopy (XPS) (kratos axis ultra DLD), Photoluminescence (PL) spectroscopy (Perkin Elmer LS 55 Flouroscence spectrophotometer), Time-resolved flouroscence spectroscopy using (Edinburgh FL 920 Flourscence lifetime spectrometer), and Thermogravometric analysis (TGA) (Perkin Elmer STA 6000).

\section{Results and discussion}

The recorded XRD pattern of silver nanowires in Fig. 1 shows that there are five diffraction peaks, which agree well with the (111), (200), (220), (311), and (222) diffractions of face-centered cubic (FCC) silver nanostructure and are comparable with the standard powder diffraction card of Joint Committee on Powder Diffraction Standards (JCPDS File No. 04-0783 from ASTM). The calculated lattice constant according to the spacing distance $\mathrm{d}(0.235 \mathrm{~nm})$ of the $\{111\}$ plane is $0.4083 \mathrm{~nm}$, and the same has been calculated using the equation: $1 / \mathrm{dg}^{2}=\left(h^{2}+k^{2}+l^{2}\right) / a^{2}$ (Maillard et al. 2002). This is in agreement with the literature value of $0.4086 \mathrm{~nm}$. It indicates that the high-purity FCC silver nanowires could be prepared by this method.

Topography of the silver nanowires has been analyzed by SEM. Figure 2 shows SEM micrographs of silver nanowires and these micrographs reveal that the products consist of a large amount of silver nanowires with lengths in the range of several tens of micrometer, while their diameters are in the range of tens of nanometers. The aspect ratio of the corresponding nanowires is very high. Purity of the silver nanowires synthesized using this method with optimal conditions of $\mathrm{NaCl}, \mathrm{PVP}, \mathrm{AgNO}_{3}$, and heating time is more than $95 \%$, and the same is revealed by TEM micrograph, Fig. $5 a$, b.

FESEM micrograph in Fig. 3 reveals that at higher concentration of $\mathrm{NaCl}(10 \mathrm{mM})$, besides one-dimensional (nanorods and nanowires) products, amorphous silver chloride particles also appeared.

$\mathrm{X}$-ray energy dispersive microanalysis of the silver nanostructures in Fig. 4 shows that the sample was essentially pure silver, and almost no oxygen was present. Recorded spectra indicate that silver content is $51.58 \mathrm{wt} \%$ whereas that of chlorine is $48.42 \mathrm{wt} \%$.

Morphology of silver nanowires has been analyzed using TEM. Morphology from Fig. 5a clearly indicates that

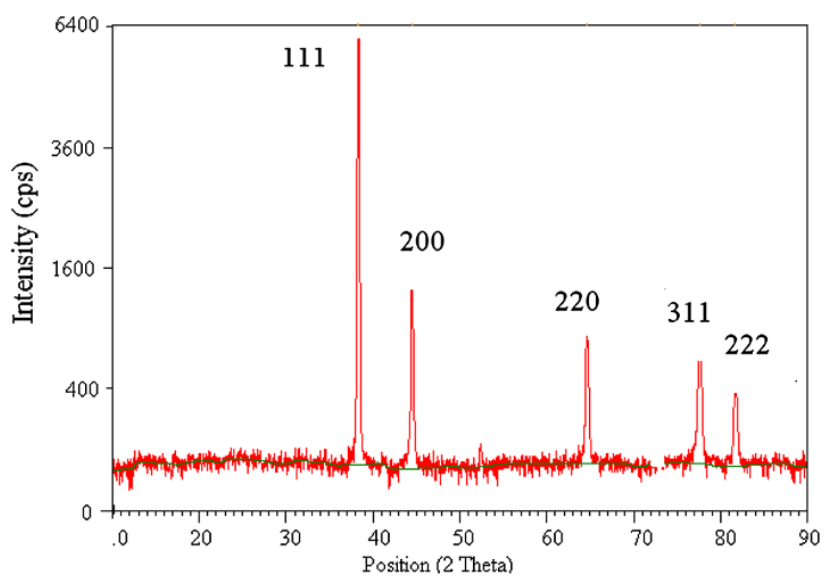

Fig. 1 XRD pattern of silver nanowires 

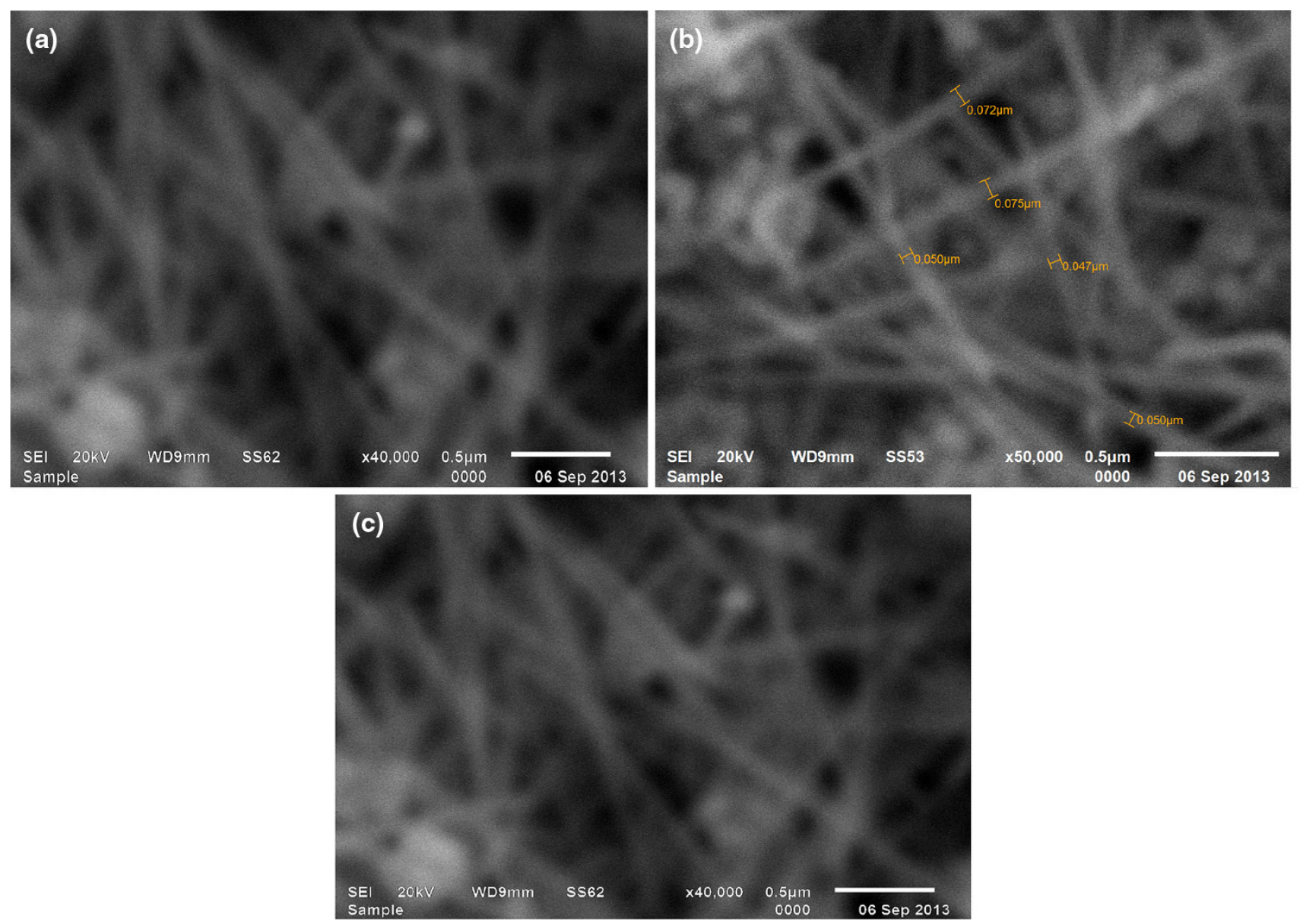

Fig. 2 SEM micrograph of silver nanowires

nanowires are of micrometer range in length and nanometer range in diameter. Figure $5 \mathrm{~b}$ shows the micrograph of single nanowire and Fig. $5 \mathrm{c}$ that of silver nanowires at high concentration of $\mathrm{NaCl}(10 \mathrm{mM})$.

Figure 6 shows the UV-visible absorption spectrum taken from aqueous suspension of the as-obtained product. The broad peak centered at $400 \mathrm{~nm}$ in the as-prepared sample is attributed to the transverse plasmon excitation of silver nanowires. The shoulder peak at $352 \mathrm{~nm}$ is attributed to the plasmon response of bulk silver which is commonly observed for silver nanowires (Gao et al. 2005a; Korte et al. 2008; Wang et al. 2005; Sun et al. 2002b).

Figure 7 shows the FTIR spectra of silver nanowires with absorption peak located at around $1,291 \mathrm{~cm}^{-1}$ which can be assigned to the stretching vibration of $-\mathrm{C}-\mathrm{N}-$. The peaks at 1,659 and $2,954 \mathrm{~cm}^{-1}$ are due to $-\mathrm{C}=\mathrm{O}$ (carbonyl group) and $-\mathrm{C}-\mathrm{H}$ stretching vibrations, respectively. In addition, there is a broad peak located at around $3,438 \mathrm{~cm}^{-1}$, which can be assigned to the $\mathrm{O}-\mathrm{H}$ stretching vibration, indicating the presence of hydroxyl groups (Wang et al. 1982). The absorption peaks located at around $1,645 \mathrm{~cm}^{-1}$ can be assigned to the stretching vibration of $-\mathrm{C}=\mathrm{O}$. The weak peaks at 2,923 and $2,848 \mathrm{~cm}^{-1}$ are due to $-\mathrm{CH}_{3}$ and $-\mathrm{CH}_{2}-$ stretching vibrations. The absorption peak at about $3,394 \mathrm{~cm}^{-1}$ can be assigned to the stretching vibration of O$\mathrm{H}$. The carbonyl absorption peak of nano-Ag/PVP composites compared with pure PVP is red-shifted from 1,659 to $1,645 \mathrm{~cm}^{-1}$; this is mainly because the oxygen atoms of the carbonyl get part of the electrons in silver nanowires. Therefore, one can conclude that there is a certain interaction between silver nanowires and carbonyl in PVP. The conclusions are consistent in that there is an adsorption interaction between PVP and nanosilver atoms, which is described by Yang and $\mathrm{Lu}$ (2005).

Figure 8 shows the Raman spectra of the AgNWs, and the appearance of $\mathrm{Ag}-\mathrm{O}$ stretching vibrations at $237 \mathrm{~cm}^{-1}$ confirms that the PVP molecule is coordinated to the Ag surface through the non-bonding electrons of the oxygen atom in carbonyl, which is consistent with the results of XPS. Meanwhile, there appears very significantly enhanced Raman peaks at $1,605 \mathrm{~cm}^{-1}$, which can be assigned to the stretching vibration of $\mathrm{C}=\mathrm{O}$, indicating that PVP molecules have interaction with silver nanowires mainly through oxygen of $\mathrm{C}=\mathrm{O}$. The nearer the compounds of 

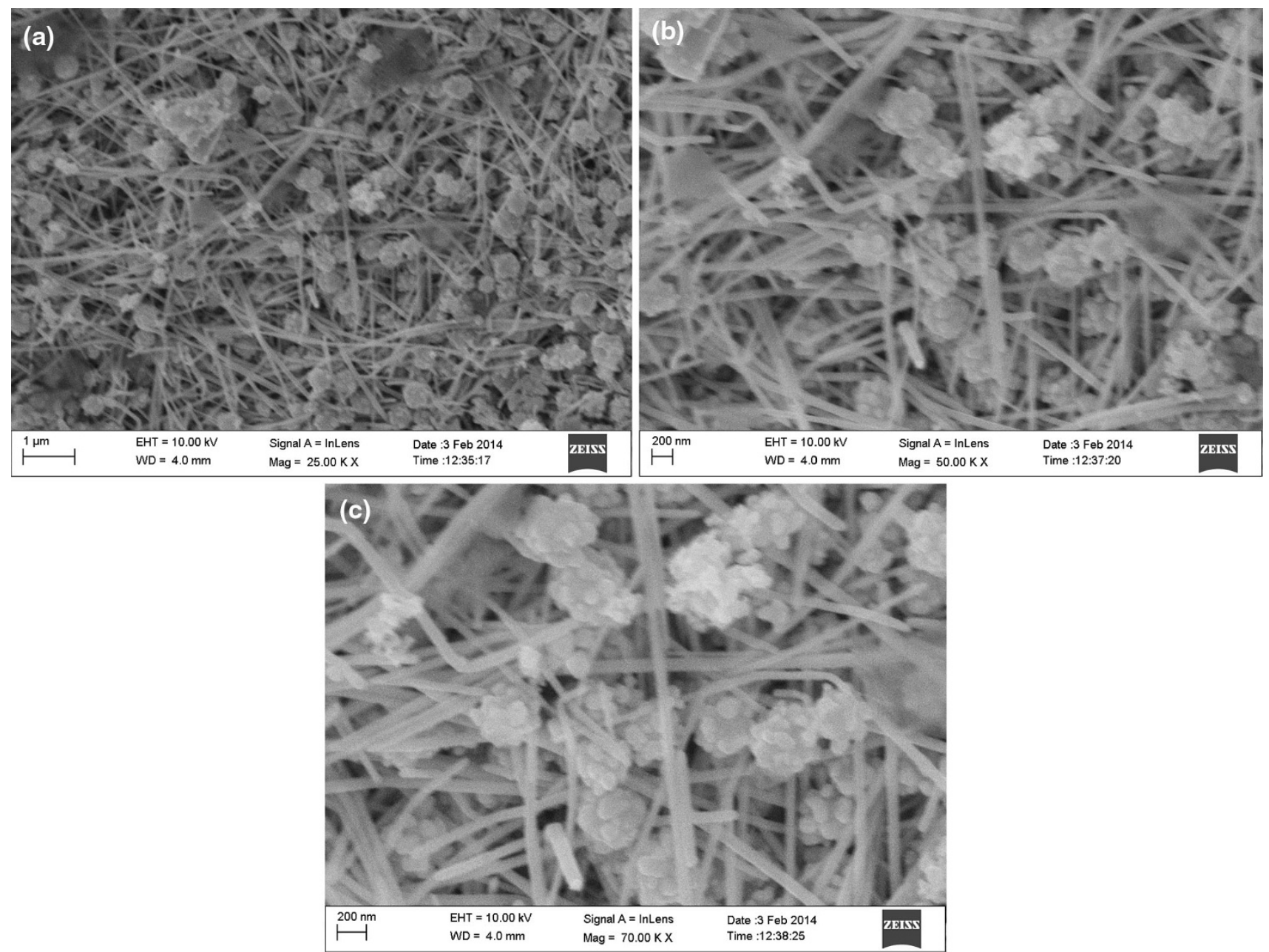

Fig. 3 FESEM micrograph of silver nanostructures at high concentration of $\mathrm{NaCl}(10 \mathrm{mM})$

Fig. 4 EDS of silver nanostructures at high concentration of $\mathrm{NaCl}(10 \mathrm{mM})$

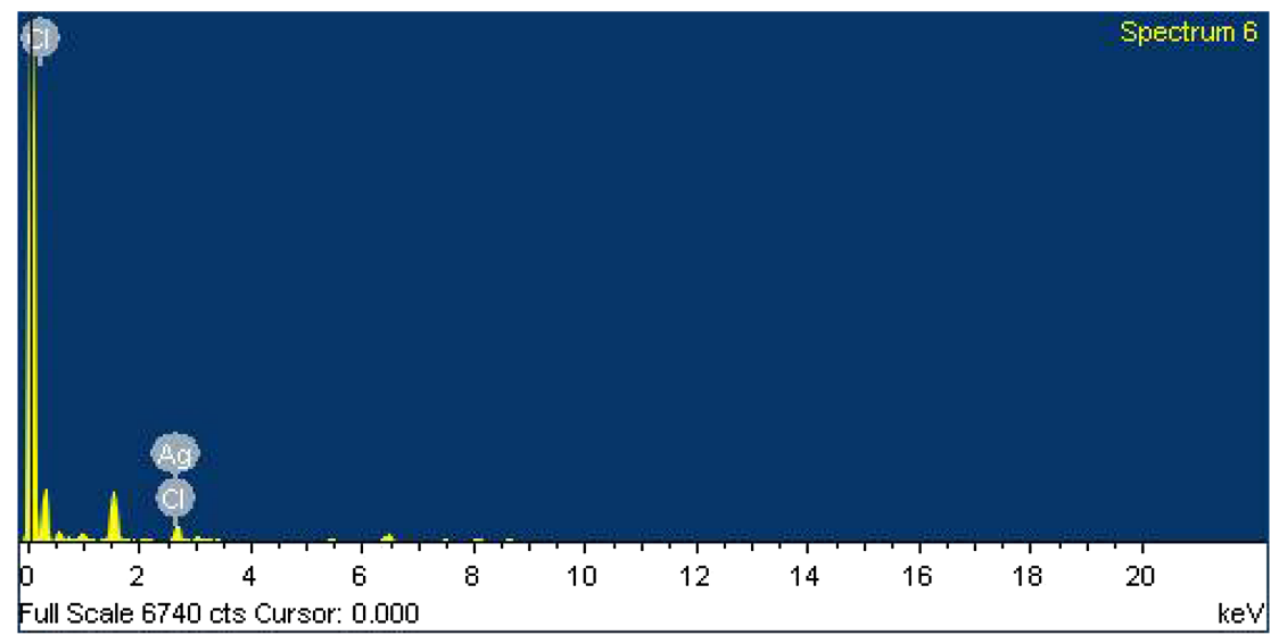

the functional group and the silver nanowires to each other, the more significantly enhanced will be their Raman spectra. The significant enhancement of the signal at $2,936 \mathrm{~cm}^{-1}$ (asymmetric stretching vibration of $\mathrm{CH}_{2}$ in the skeletal chain of PVP) reveals that the $\mathrm{CH}_{2}$ chain is close to the surface of Ag nanowires.

To further investigate the interaction between PVP and Ag nanowires, XPS was performed on the purified Ag 

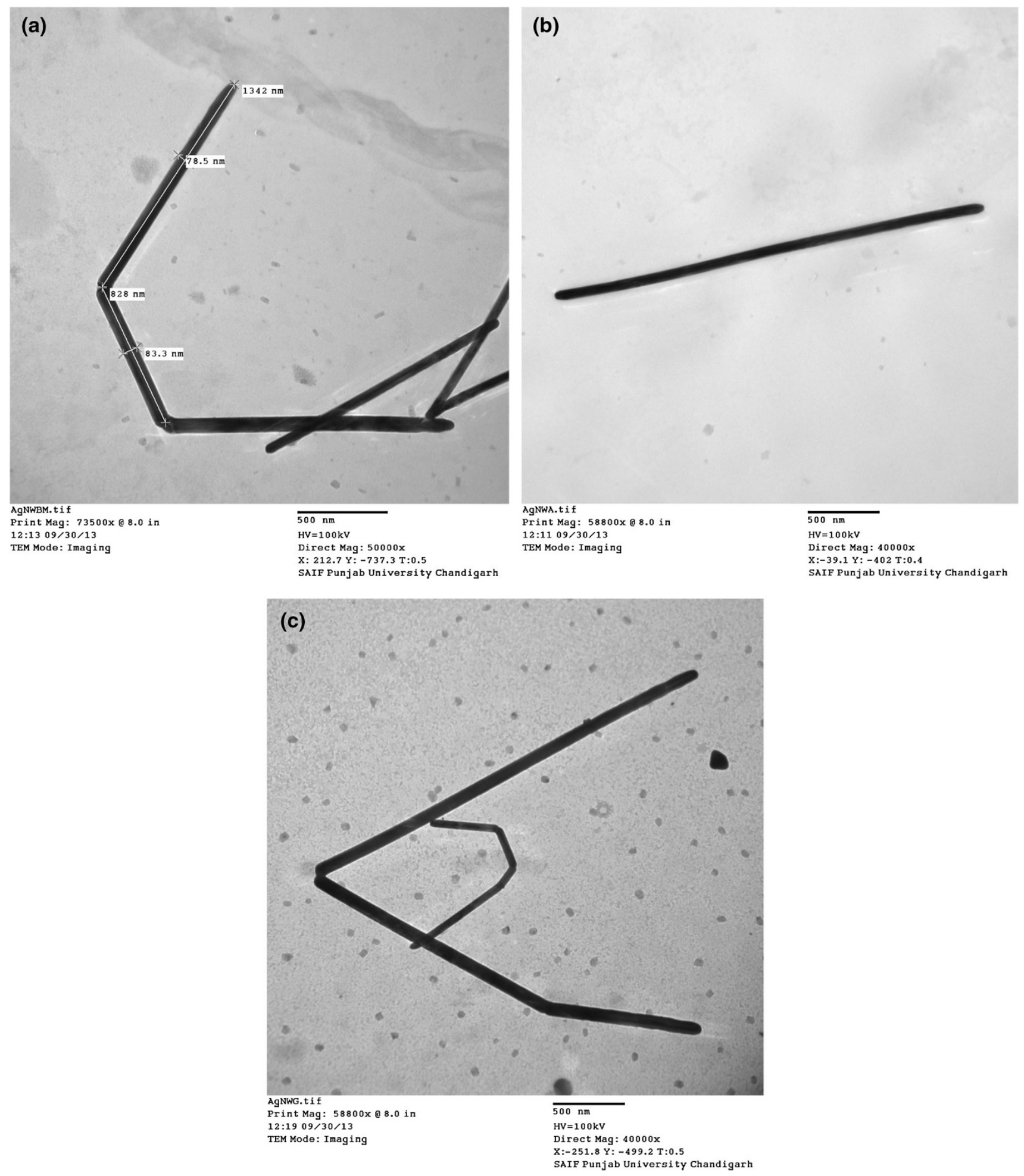

Fig. 5 TEM micrograph of silver nanowires

nanowires. Figure 9a shows the XPS spectra of the purified Ag nanowires. In addition to Ag element on the spectrum, there are peaks of $\mathrm{C}, \mathrm{O}$, and $\mathrm{N}$ elements. Figure $9 \mathrm{~b}$ shows the XPS spectrum in the region of $\mathrm{Ag} \mathrm{3d}$ of the $\mathrm{Ag}$ nanowires. It can be seen that two peaks occur at 367.35 and $373.35 \mathrm{eV}$, corresponding to $\mathrm{Ag} 3 \mathrm{~d}_{5 / 2}$ and $3 \mathrm{~d}_{3 / 2}$ binding energies, respectively. In comparison with $\mathrm{Ag}^{0}$ (368.3 and $374.3 \mathrm{eV}$ ), the peaks shift to lower binding energy, indicating that the chemical environment around $\mathrm{Ag}$ atoms has changed. Figure 9c shows that the chemical environment 


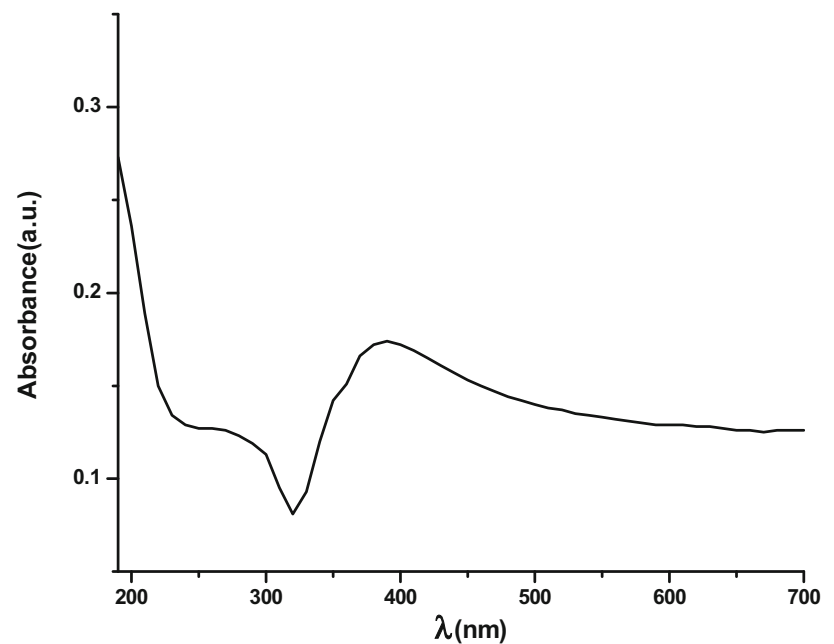

Fig. 6 UV-visible absorption spectra of silver nanowires

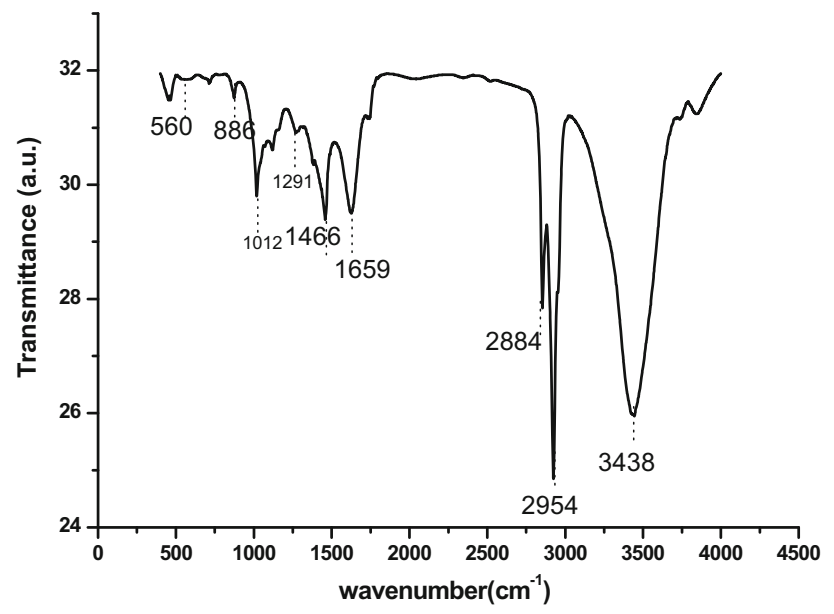

Fig. 7 FTIR spectra of silver nanowires

around $\mathrm{C}$ element has not changed. Figure $9 \mathrm{~d}$ shows the XPS spectra of $\mathrm{O} 1 \mathrm{~s}$ of the purified $\mathrm{Ag}$ nanowires. It is observed from Fig. 8d that, in the case of PVP-capped Ag nanowires, the peak attributed to $\mathrm{O} 1 \mathrm{~s}$ is shifted toward higher binding energy compared with pure PVP. The large shift $(0.9 \mathrm{eV})$ of the $\mathrm{O} 1 \mathrm{~s}$ binding energy originates from the weakness of electron density around $\mathrm{O}$ atoms in the carbonyl group of PVP, implying that the surface Ag atoms strongly coordinate with $\mathrm{O}$ atoms in PVP (Huang et al. 1996). Thus, it is probably the strong Ag: O coordination that restricts the radial growth of the $\mathrm{Ag}$ nanowires along the (100) direction.

TGA analysis of silver nanowires done in air flow is shown in Fig. 10. It can be seen from the graph in the figure that weight loss from the beginning up to $280{ }^{\circ} \mathrm{C}$ is very less. There is a larger mass loss after $280^{\circ} \mathrm{C}$. The weight loss from 280 to $380{ }^{\circ} \mathrm{C}$ is attributed to the decomposition of $-\mathrm{CH}_{3}$ side chain in PVP. As the

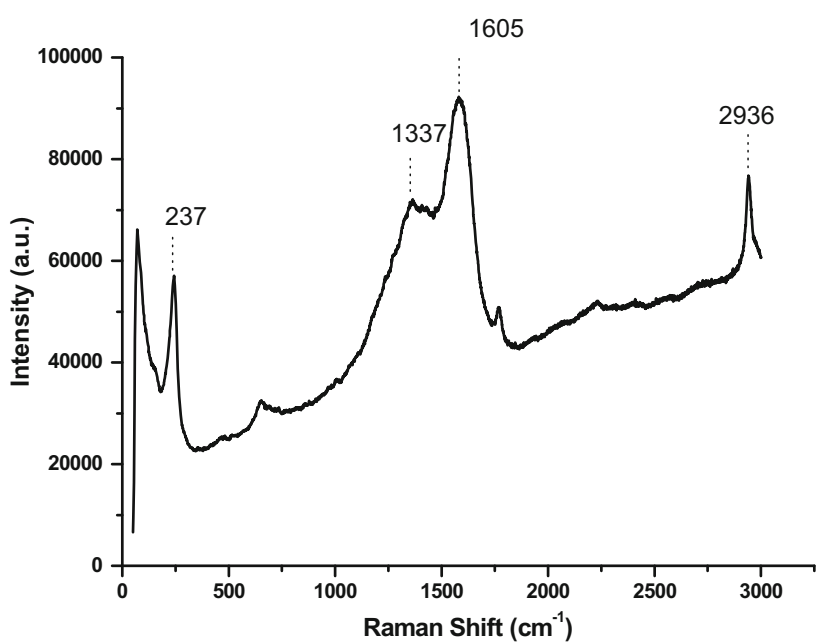

Fig. 8 Raman spectra of silver nanowires

temperature continues to rise, the carbon rings begin to decompose. The weight loss after $790{ }^{\circ} \mathrm{C}$ is mainly due to the decomposition of the main chain of PVP, clearly indicating that PVPs decompose completely after $790{ }^{\circ} \mathrm{C}$. From the DSC curves, one can see there is an endothermic peak at $48.7^{\circ} \mathrm{C}$, which can be attributed to the evaporation of ethanol and adsorbed water. At $449.5 .5^{\circ} \mathrm{C}$, there is an exothermic peak, which is mainly due to the decomposition of the main chain of PVP.

Figure 11 shows the room-temperature-recorded steadystate PL spectra of AgNWs. The variations of PL emission from the silver nanoclusters dispersed in water are recorded at room temperature at the excitation wavelengths of 300 and $400 \mathrm{~nm}$. The blue-green PL emission is observed from the prepared samples, and these emissions are assigned to radiative recombination of Fermi level electrons and sp- or d-band holes.

When the samples are exposed to high-intensity laser radiations, the electrons are raised from the valence band to the excited states, and then these excited electrons return back to valence band with the emission of characteristic luminescent radiations. The intensity ' $I$ ' of the luminescent radiation at any time, $\mathrm{t}$, is given by following equation:

$I=I_{0} \mathrm{e}^{-p t}$

where $I_{0}$ is the intensity of radiation at cut-off position, and the constant $p=1 / \tau$ is the transition probability of the corresponding radiative transition. Here, $\tau$ is the excited state lifetime, i.e., the time spent by electron in the trapping state before recombination. From the slope of the $\ln (I)$ vs. $t$, one can easily calculate transition probability. Figure 12 shows time-resolved decay curve recorded for silver nanowires.The $\operatorname{Ln}(I)$ vs. $t$ graphs were plotted for all the recorded decay curves. These $\operatorname{Ln}(I)$ vs. $t$ graphs did not show linear relationship because of the superposition of a 
(a)

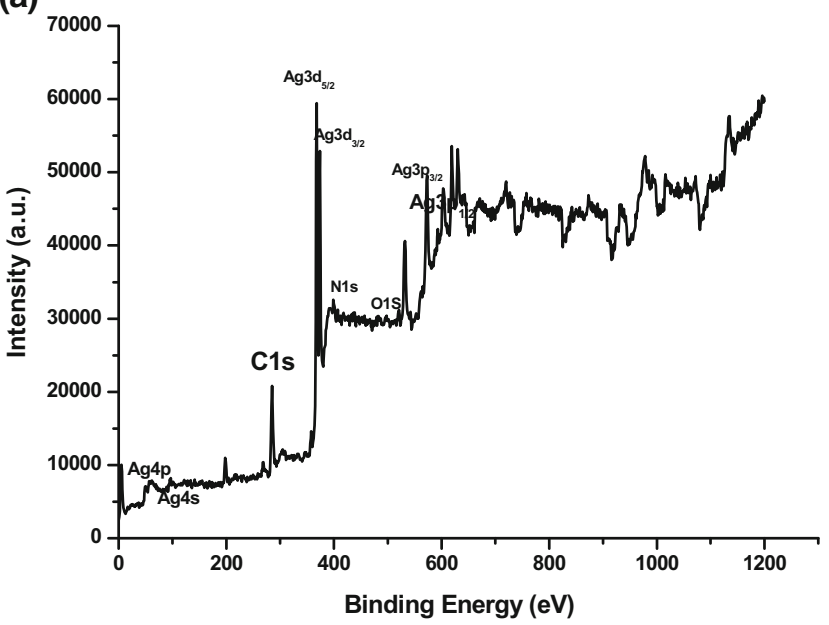

(c)

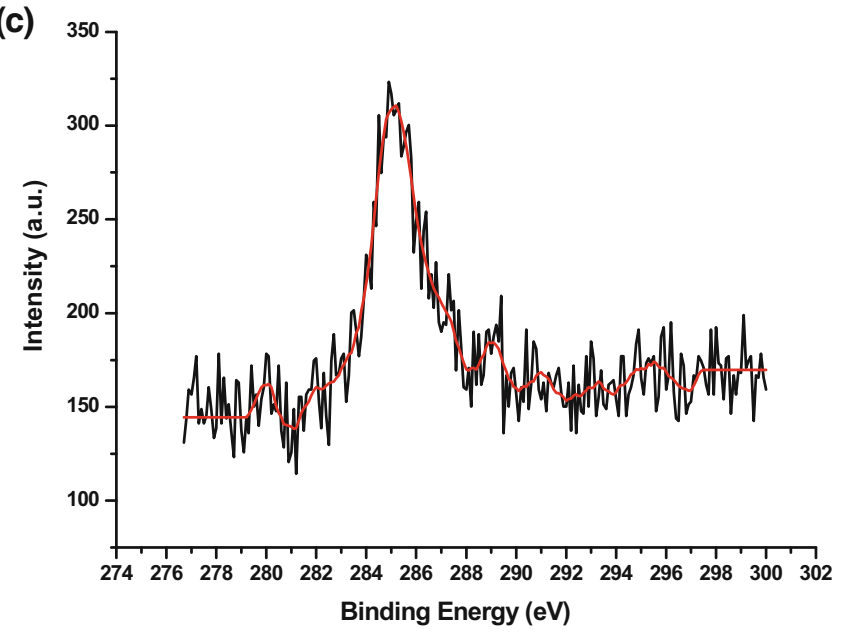

(b)

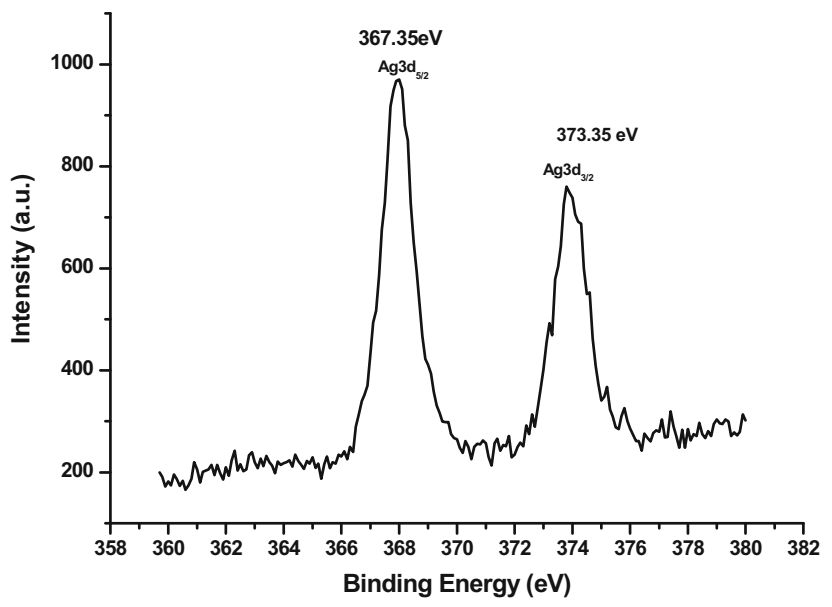

(d)

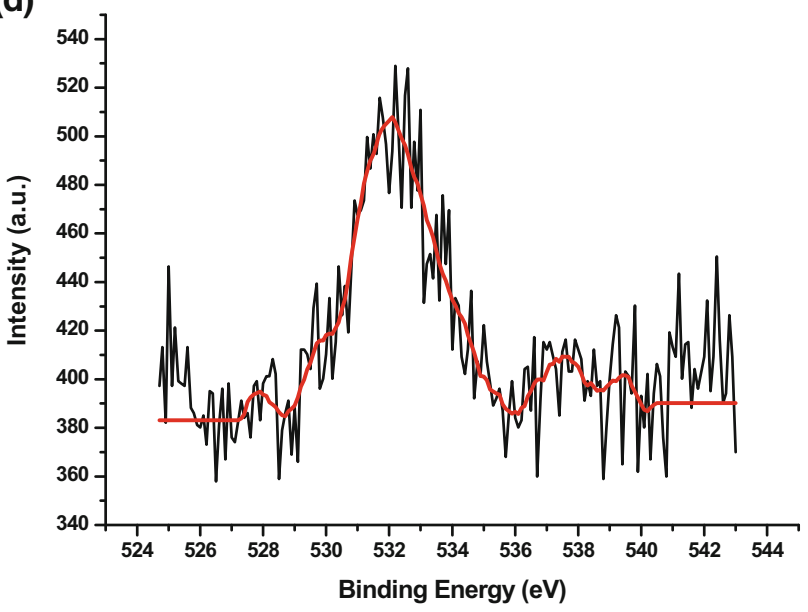

Fig. 9 a XPS spectra of silver nanowires, b XPS spectra of Ag3d purified silver nanowires, $\mathbf{c}$ XPS spectra of C1s of purified silver nanowires, d XPS spectra of $\mathrm{O} 1 \mathrm{~s}$ of purified silver nanowires

number of exponential decays. These graphs were peeledoff into maximum of three components using peeling-off method of Singh et al. 2009. The slope of each component gives the value of transition probability $(p)$ and hence the

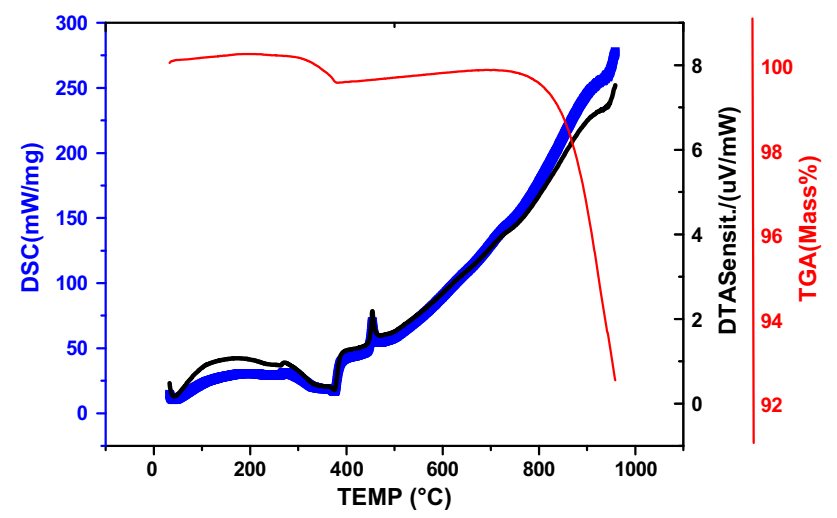

Fig. 10 TGA, DSC, and DTA analyses of silver nanowires excited state lifetime ' $\tau$ '. Trap-depth values ' $E$ ' had been calculated using Boltzmann Eq. (2). All these values are listed in Table 1.

$p=S \mathrm{e}^{-E / k T}$

where $p$ is transition probability; $S$ ie the escape frequency factor $\left(\sim 10^{9} \mathrm{~s}^{-1}\right) ; k$ is the Boltzmann's constant and $\mathrm{T}$ is the absolute temperature. Distribution of traps within the phosphor bandgap can be explained with the help of decay constant values. The decay constant value ' $b$ ' has been calculated using the following equation:

$I=I_{\mathrm{o}} t^{-b}$

Such types of decay curves due to the superposition of a number of exponential decays are popularly known as hyperbolic decay curves or multiexponential decay curves. If the value of $b$ is unity, one can say that the distribution of trapping states is uniform; otherwise, it is said to be nonuniform. The $\operatorname{Ln}(I)$ vs. $\operatorname{Ln}(t)$ graph was plotted 

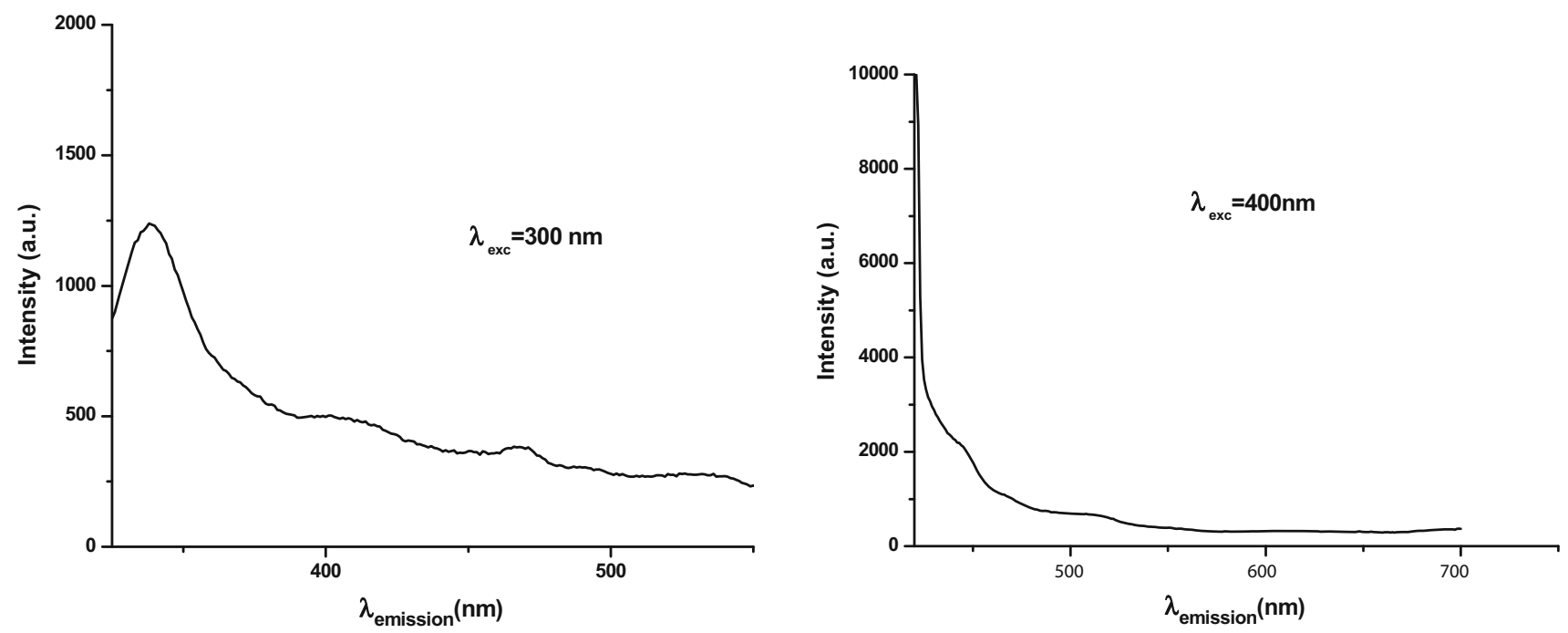

Fig. 11 PL spectra of silver nanowires at excitation wavelengths of 300 and $400 \mathrm{~nm}$

corresponding to emission wavelength of $339.6 \mathrm{~nm}$, and the decay constant ' $b$ ' value has been calculated from the slope of these graphs according to Eq. (3). For AgNWs, this value is calculated to be 0.97018 which means the distribution of traps within the sample is nonuniform. In terms of lifetime values so calculated, one can calculate the oscillator strengths and dipole moments of the radiative transitions using the well-known equations. Electromagnetic radiation interacts with an electronic center through the electric or magnetic field of the radiation. The oscillator strength, $\mathrm{f}$, of a transition is a dimensionless quantity that is useful for comparing different radiative transitions. Classically, oscillator strength is used as a statistical weight indicating the relative number of oscillators bound at each resonant

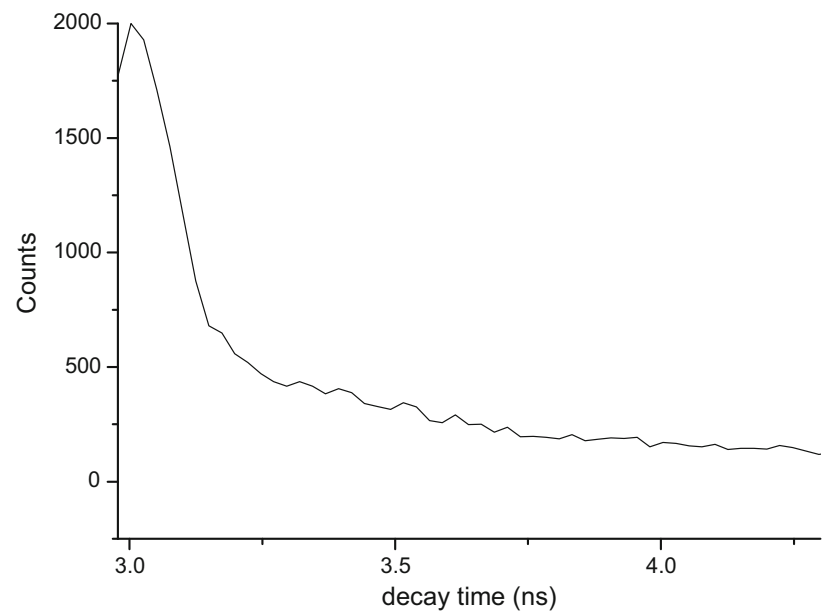

Fig. 12 Time-resolved decay curve for silver nanowires at room temperature frequency. In quantum mechanics, oscillator strength is used as a measure of relative strength of the electronic transitions within atomic and molecular systems. Oscillator strength is particularly useful as a technique for comparing transition "strengths" between different types of quantum mechanical systems. The lifetime values may also be used to calculate Einstein's spontaneous $(A)$, stimulated emission $(B)$ coefficients, and dipole moment $\mu$ of the radiative transtion. Moreover, the oscillator strength along with absorption coefficients is often used as a method for calculating the concentration of impurities in a host. The oscillator strength of electric dipole-allowed transitions (Wu et al. 2001) is given by the

Table 1 Transition probability, excited state lifetime, trap depth, and other important optical parameters of silver nanowires

\begin{tabular}{lll}
\hline$p_{1}\left(\mathrm{~ns}^{-1}\right)$ & $p_{2}\left(\mathrm{~ns}^{-1}\right)$ & $p_{3}\left(\mathrm{~ns}^{-1}\right)$ \\
6.70298 & 1.35509 & 0.23742 \\
$\tau_{1}(\mathrm{~ns})$ & $\tau_{2}(\mathrm{~ns})$ & $\tau_{3}(\mathrm{~ns})$ \\
0.149 & 0.7379 & 4.212 \\
$E_{1}(\mathrm{eV})$ & $E_{2}(\mathrm{eV})$ & $E_{3}(\mathrm{eV})$ \\
0.21 & 0.2279 & 0.2473 \\
$I_{\mathrm{c} 1}\left(10^{-10} \mathrm{~m}^{2} \mathrm{~s}^{-1}\right)$ & $I_{\mathrm{c} 2}\left(10^{-10} \mathrm{~m}^{2} \mathrm{~s}^{-1}\right)$ & $I_{\mathrm{c} 3}\left(10^{-10} \mathrm{~m}^{2} \mathrm{~s}^{-1}\right)$ \\
$306,483.01$ & $62,156.83$ & $10,890.25$ \\
$\mu_{1}\left(\times 10^{-31} \mathrm{~cm}\right)$ & $\mu_{2}\left(\times 10^{-31} \mathrm{~cm}^{-1}\right)$ & $\mu_{3}\left(\times 10^{-31} \mathrm{~cm}\right)$ \\
$8,004.16$ & $3,599.66$ & $1,506.41$ \\
$f_{1}\left(\times 10^{-5}\right)$ & $f_{2}\left(\times 10^{-5}\right)$ & $f_{3}\left(\times 10^{-5}\right)$ \\
$427,707.85$ & $86,466.41$ & $15,149.44$ \\
$B_{1}\left[\times 10^{18} \mathrm{~m}^{3}(\mathrm{rad} / \mathrm{s}) / \mathrm{Js}\right]$ & $B_{2}\left[\times 10^{18} \mathrm{~m}^{3}\right.$ & $B_{3}\left[\times 10^{18} \mathrm{~m}^{3}\right.$ \\
& $(\mathrm{rad} / \mathrm{s}) / \mathrm{Js}]$ & $(\mathrm{rad} / \mathrm{s}) / \mathrm{Js}]$ \\
$516,966.21$ & $104,587.11$ & $18,311.2$ \\
\hline
\end{tabular}

It is evident from the above data that the excited state lifetime for silver nanowires varies from 0.149 ns to $4.212 \mathrm{~ns}$ 
$f_{\text {ed }}(v)=15 \times 10^{4} \lambda^{2} \frac{9}{\left(n^{2}+2\right)^{2} n} \times p$

following relation (4):

where $\lambda$ is the emission wavelength, $p$ is the transition probability of the corresponding radiative transition, and ' $n$ ' is the index of refraction of the material which is 1.45 in the case of AgNWs. The spontaneous radiative lifetime is seen to be related in a simple way to the integrated cross section of the transition (Wu et al. 2001) by the following relation:

$I_{\mathrm{cs}}=\frac{\lambda^{2}}{8 \pi} \times p$

$|\mu|=\left(\frac{3 h \varepsilon_{0} \lambda^{3}}{16 \pi^{3} n} \times p\right)^{\frac{1}{2}}$

The corresponding dipole-moment (Wu et al. 2001) is given by the relation:

$B=\frac{2 \pi^{2} \mu^{2}}{3 n^{2} \varepsilon_{0} h^{2}}$

Dipole-moment values of AgNWs were calculated using the above equation in terms of emission wavelength, index of refraction, and the excited state lifetime values. Einstein's stimulated coefficient is given by the following relation:

where ' $\mu$ ' is the dipole moment of transitions from the shallow trapping states of AgNWs ' $\varepsilon_{0}$ ' is the absolute permittivity of free space, ' $n$ ' is the index of refraction, and ' $h$ ' is Planck's constant

\section{Conclusions}

In conclusion, $\mathrm{Ag}$ nanowires within a very short time of few minutes have been successfully synthesized by means of microwave-polyol method. The shapes and sizes of Ag nanostructures depend strongly on such experimental parameters as concentrations of $\mathrm{AgNO}_{3}, \mathrm{PVP}, \mathrm{NaCl}$, and that of heating time. At low concentration of PVP $(50 \mathrm{mM})$, medium concentration of $\mathrm{NaCl}(3 \mathrm{mM})$ and heating time of $3 \mathrm{~min}$ are favorable for the formation of $\mathrm{Ag}$ nanorods and nanowires. The synthesis of $\mathrm{Ag}$ nanowires in the presence of $\mathrm{NaCl}$ is a new point of highlight of this study. The advantages here are simple equipment and process, the much cheaper $\mathrm{NaCl}$ compared to $\mathrm{H}_{2} \mathrm{PtCl}_{6}$ used in other previous publications. The XPS investigation shows that the PVP molecules are absorbed on the surface of the $\mathrm{Ag}$ nanowires through $\mathrm{Ag}: \mathrm{O}$ coordination. It is reasonable to deduce from the above results that the interaction between PVP molecules and $\mathrm{Ag}$ atoms on $\{100\}$ planes is stronger than that on $\{111\}$, which leads to the anisotropic growth along the $\langle 110\rangle$ direction, while the growth rate along the $\langle 100\rangle$ direction is reduced. The results from Raman spectra indicate the skeleton chain of the PVP molecules is almost lying on the surface of the Ag nanowire, and the pyrrolidone ring might be tilted on the surface of Ag nanowires. These results show that the PVP molecules are used both as a protecting agent and a structure-directing agent for the growth of $\mathrm{Ag}$ nanowires. Further lifetime values calculated from decay curve have been used for the calculation of other important optical parameters of optoelectronic industrial interest like trapdepths, transition probabilities, oscillator strengths, Einstein's coefficients, integrated cross-sections, etc., which will be beneficial for optical calibration curves and detailed phosphor characterization for the twenty-first century industrial applications.

Open Access This article is distributed under the terms of the Creative Commons Attribution License which permits any use, distribution, and reproduction in any medium, provided the original author(s) and the source are credited.

\section{References}

Gao Y, Jiang P, Liu DF, Yuan HJ, Yan XQ, Zhou ZP, Wang JX, Song L, Liu LF, Zhou WY, Wang G, Wang CY, Xie SS (2004) Evidence for the monolayer assembly of poly(vinylpyrrolidone) on the surfaces of silver nanowires. J Phys Chem B 108(34):12877-12881

Gao X, Gu G, Hu Z, Guo Y, Fu X, Song J (2005a) A simple method for preparationof silver dendrites. Colloids Surf A 254:57-61

Gao Y, Jiang P, Song L, Liu L, Yan X, Zhou Z, Liu D, Wang J, Yuan H, Zhang Z, Zhao X, Dou X, Zhou W, Wang G, Xie S (2005b) Growth mechanism of silver nanowires synthesized by polyvinylpyrrolidone-assisted polyol reduction. J Phys D Appl Phys 38:1061

Huang HH, Ni XP, Loy GL, Chew CH, Tan KL, Loh FC, Deng JF, Xu GQ (1996) Photochemical formation of silver nanoparticles in poly (N vinylpyrrolidone). Langmuir 12:909-912

Kim SH, Choi BS, Kang KK, Choi YS, Yang SI (2007) Low temperature synthesis and growth mechanism of Ag nanowires. J Alloys Compd 433:261-264

Korte KE, Skrabalak SE, Xia Y (2008) Rapid synthesis of silver nanowires through aCuCl- or $\mathrm{CuCl} 2$-mediated polyol process. J Mater Chem 18:437-441

Maillard M, Giorgio S, Pileni MP (2002) Silver nanodisks. Adv Mater 14:1084-1086

Mazur M (2004) Electrochemically prepared silver nanoflakes and nanowires. Electrochem Commun 6:400-403

Singh K, Verma NK, Bhatti HS (2009) Photoluminescence studies of Cd1-xZnxS nanocrystals. Phys B 404:300-304

Sun Y, Yin Y, Mayers BT, Herricks T, Xia Y (2002a) Uniform silver nanowires synthesis by reducing $\mathrm{AgNO}_{3}$ with ethylene glycol in presence of seeds and poly (vinyl pyrrolidine). Chem Mater $14: 4736-4745$

Sun YG, Xia YN (2002b) Large scale synthesis of silver nanowires through a soft self seeding, polyol process. Adver Mater 14:833-837

Sun Y, Xu FQ, Li ZM, Zhang WH (2005) Cyclic voltammetry for the fabrication of high dense silver nanowire array with assistance of AAO template. Mater Chem Phys 90:69-72 
Tsuji M, Hashimoto M, Nishizawa Y (2005) Microwave-assisted synthesis of metallic nanostructures in solution. Chemistry 11:440-452

Wang ZM, He XX, Sun DQ (1982) Practical infrared spectroscopy petroleum. Industry Press, Beijing, pp 164-166

Wang Z, Liu J, Chen X, Wan J, Qian Y (2005) A simple hydrothermal route to large-scale synthesis of uniform silver nanowires. Chem Eur J 11:160-163

Wu XL, Siu GG, Fu CL, Ong CH (2001) Photoluminescence and cathodoluminescence studies of stoichiometric and oxygendeficient ZnO films. Appl Phys Lett 78:2285-2287

Xu J, Hu J, Peng CJ, Liu HL, Hu Y (2006) A simple approach to the synthesis of silver nanowires by hydrothermal process in the presence of gemini surfactant. J Colloid Interface Sci 298:689-693

Yang XM, Lu Y (2005) Preparation of polypyrrole-coated silver nanoparticles by one step UV-induced polymerization. Mater Lett 59:2484-2487

Yang Y, Hu Y, Xiong X, Qin Y (2013) Impact of microwave power on the preparation of silver nanowires via a microwave-assisted method. RSC Adv 3:8431-8436

Zou K, Zhang XH, Duan XF, Meng XM, Wu SK (2004) Seedmediated synthesis of silver nanostructures and polymers/silver nanocables by UV irradiations J Cryst Growth 273:285-291 